\title{
An approach for reliability-based robust design optimisation of angle-ply composites
}

\author{
Carlos Conceição António ${ }^{\mathrm{a}, *}$, Luísa N. Hoffbauer ${ }^{\mathrm{b}}$ \\ ${ }^{a}$ Faculdade de Engenharia, University of Porto, Rua Dr. Roberto Frias, 4200-465 Porto, Portugal \\ ${ }^{\mathrm{b}}$ Instituto Superior de Engenharia do Porto, Polytechnic School of Porto, Porto, Portugal
}

\begin{abstract}
Variations of manufacturing process parameters and environmental aspects may affect the quality and performance of composite materials, which consequently affects their structural behaviour. Reliability- based design optimisation (RBDO) and robust design optimisation (RDO) searches for safe structural sys- tems with minimal variability of response when subjected to uncertainties in material design parameters. An approach that simultaneously considers reliability and robustness is proposed in this paper. Depending on a given reliability index imposed on composite structures, a trade-off is established between the performance targets and robustness. Robustness is expressed in terms of the coefficient of variation of the constrained structural response weighted by its nominal value. The Pareto normed front is built and the nearest point to the origin is estimated as the best solution of the bi-objective optimisation problem.
\end{abstract}

Keywords:

Composite structures Robust design Reliability, Bi-objective optimisation

\section{Introduction}

Structural applications of composite materials have increased due to their excellent specific stiffness, low weight, and reduced energy consumption. Variations of manufacturing process parameters and environmental aspects may affect the quality and performance of the product, which consequently affects its structural behaviour. Therefore, the need for reliability analysis is increasing, and reliability-based design optimisation (RBDO) of composite structures is currently a very important area of research [1-5].

Uncertainty and sensitivity analysis are important in studying complex systems, such as composite laminated structures, for robustness assessment. Specifically, uncertainty analysis refers to the determination of the uncertainty in the response as a result of uncertainties in random variables, and sensitivity analysis refers to the determination of the contributions of individual uncertainties of random variables to the uncertainty in response results. A number of approaches to uncertainty and sensitivity analysis have been developed, including differential analysis, response surface methodology, Monte Carlo analysis, and variance decomposition procedures. Reviews of these methodologies are available in the bibliography [6-9].

Robust design optimisation (RDO) of composite structures is an-

other emerging area of research that is thought of as an alternative

design to RBDO. Indeed, the principal objective of robust design is to improve product quality by minimising the uncertainty effects or stabilising variations in structural response without eliminating their causes. Robust design optimisation (RDO) of composite structures under probabilistic constraints is a very important field due to uncertainties associated with physical properties of fibre-reinforced composites [10].

Mixed formulations of RBDO and RDO appear today as a new emerging branch of reliability and robustness research areas [11]. An approach that simultaneously considers reliability and robustness is proposed in this paper. The trade-off between the performance target, depending on a given reliability index imposed on composite structures, against robustness, expressed in terms ofthe coefficient of variation of the constrained structural response weighted by its nominal value, is searched. The Pareto normed front is built, and the point closest to the origin is estimated, as the best solution of the bi-objective optimisation problem.

\section{Robust design optimisation}

In reliability-based design optimisation (RBDO), a mean objective is minimised based on probabilistic constraints related to feasibility under uncertainty. Thus, the RBDO problem is formulated as

$$
\begin{aligned}
& \underset{\mathbf{x}, \boldsymbol{\mu}_{\pi}}{\operatorname{Minimize}} \quad F\left(\mathbf{x}, \mu_{\pi}\right) \\
& \text { Subject to } \beta_{s}(\mathbf{x}, \pi) \geq \beta_{a} \\
& \mathbf{x}^{L} \leq \mathbf{x} \leq \mathbf{x}^{U}
\end{aligned}
$$

where $F\left(\mathrm{x}, 1_{p}\right)$ is a function describing the structural performance, $\times 2 R^{n}$ is the vector of deterministic design variables, $p 2 R^{m}$ is the 
vector of random variables, and $l_{p}$ is the realisation of $p$ defined as the random variables' vector. In the formulation, $b_{s}$ is the structural

reliability index, and $b_{a}$ is the prescribed reliability index. The problem defined in Eq. (1) uses two nested optimisation loops, the outer

design optimisation loop and the inner reliability assessment loop. The latter is needed for the evaluation of the structural reliability

index. Every time the optimisation loop needs to evaluate the constraint, the inner reliability assessment loop is performed to search for the most probable failure point (MPP) in the standard normal

pace.

In robust design optimisation, the robustness of the system is searched during the optimisation process to minimise the sensitivity of the performance system function in response to variability of the material properties. A bi-objective optimisation is performed by considering the following objective functions: (a) a function describing the performance of the structural response for a prescribed reliability index of the structural system, and (b) a function describing the robustness of the system related to the variability of the structural response. The bi-objective optimisation problem can then be established as

$$
\begin{aligned}
& R O B=\frac{C \cdot V \cdot L\left(\beta_{\mathrm{a}}\right)}{\bar{L}\left(\beta_{\mathrm{a}}\right)} \text { (robustness) } \\
& \text { with } \\
& \bar{L}\left(\beta_{\mathrm{a}}\right)=\operatorname{Max}\left(L_{\mathrm{k}}, \quad k=1, \ldots, N_{p}\right)
\end{aligned}
$$

where $L_{k}$ is the $k$ th component of the load vector, Lð $b_{a}$ P $2 R$. For composite structures with linear elastic structural behaviour,

$$
C \cdot V \bar{L}\left(\beta_{a}\right)=C \cdot V \bar{R}\left(\beta_{a}\right)
$$

where $C: V: R \partial b_{a} \mathrm{P}$ is the coefficient of variation of the most critical Tsai number, $R$ corresponds to the most probable failure point (MPP) obtained from the reliability index evaluation scheme and minimisation of performance function in (3). Defining the Tsai number, $R_{k}$, as a strength/stress ratio [12], it can be introduced into the interactive quadratic failure criterion of Tsai-Wu and calculated at the $k$ th point of the structure, where the stress vector is evaluated,

$$
1-\left(F_{i j} S_{i} S_{j}\right) R_{k}^{2}+\left(F_{i} S_{i}\right) R_{k}=0
$$
by solving the equation

where $s_{i}$ are the components of the stress vector, and $F_{i j}$ and $F_{i}$ are the strength parameters associated with unidirectional reinforced

$$
\begin{array}{cc}
\underset{\mathbf{x}, \mu_{\pi}}{\operatorname{Minimize}} & \left\{F\left(\mathbf{x}, \boldsymbol{\mu}_{\pi}\right), V\left(\mathbf{x}, \mu_{\pi}\right)\right\} \\
\text { Subject to } & \beta_{s}(\mathbf{x}, \pi) \geq \beta_{a} \\
& \mathbf{x}^{L} \leq \mathbf{x} \leq \mathbf{x}^{U}
\end{array}
$$

where $V\left(x, 1_{p}\right)$ is a measure of the variation of performance. At the end of the optimisation process, the Pareto front representing

the

frontier of the trade-off between the "performance" and the "robustness" functions is obtained.

\subsection{Performance of composite structures}

The objective function describing the performance is defined as the square of the difference between the structural reliability in-

dex, $b_{s}$, and the prescribed reliability index, $b_{a}$. The design variables are the ply angle, $a$, and the load factor $k$, both of which are aggre-
gated in vector $x$. The random variables, $p$, are the elasticand

strength material properties. So, the function describing the performance of the structural system is laminate defined from the macro-mechanical point of view [12]. Since the safe region is related to $R_{k}>1$, the most critical Tsai number can be established as

$\bar{R}=\operatorname{Min}\left(R_{1}, \ldots, R_{k}, \ldots, R_{N_{s}}\right)$

where $N_{s}$ is the total number of points where the stress vector is evaluated. The location of the points where the stresses are evaluated depends on the post-processing methodology used in the structural analysis. In this approach the finite element method is used for structural analysis and the stress vector is evaluated at the Gauss points of numerical integration.

From Eq. (6), the robustness can be written as,

$$
\begin{aligned}
R O B & =\frac{C \cdot V \cdot \bar{L}\left(\beta_{a}\right)}{\bar{L}\left(\beta_{a}\right)}=\frac{C \cdot V \cdot \bar{R}\left(\beta_{a}\right)}{\bar{L}\left(\beta_{a}\right)} \\
& =\frac{\sqrt{\operatorname{var}\left(\bar{R}\left(\beta_{a}\right)\right)}}{\bar{L}\left(\beta_{a} \bar{R}\left(\beta_{a}\right)\right)}
\end{aligned}
$$

$$
F\left(\lambda, a, \mu_{\pi}\right)=\left[\beta_{s}\left(\lambda, a, \mu_{\pi}\right)-\beta_{a}\right]^{2}
$$


where $l_{p}$ is the realisation of random variables. The vector of applied loads is defined as $\mathrm{L}^{1 / 4} \quad k \mathrm{~L}^{\text {ref }}$, where $\mathrm{L}^{\text {ref }}$ is the reference load

vector. The reliability index, $b_{a}$, is the target for the structural reliability index.

\subsection{Formulation for robustness}

The fundamental objective of robust design is to improve the

structural safety and to stabilise response performances by minimising the effects of the propagation of uncertainties. Although some methods are proposed in the literature for robustness assessment, their properties, in terms of accuracy and efficiency, are not yet fully known. However, the robustness of a perfor- mance is associated with the dispersion around its mean. The two most important robustness measures are the traditional var- iance and the recently proposed percentile difference [8]. An alter- native way to define robustness is proposed here. Since, from minimisation of the performance function defined in Eq. (3), the maximum load applied to the structure is obtained for $b_{s}=b_{a}$, deviations from that critical load can be associated with performance variations. Then, in this work, the robustness of the structural system is defined using the coefficient of variation, C:V:L $\bar{\varnothing} b_{\mathrm{a}} \mathrm{P}$, of the maximum load, $L \bar{\varnothing} b_{a} \mathrm{P}$, applied to the structure weighted by

its nominal value:

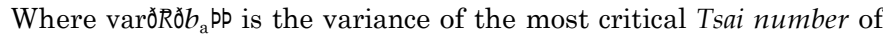
the composite structure. Since the product $L \delta b_{a} \mathrm{P} R \partial b_{a} \mathrm{P}$ is equal to the reference load for composite structures with linear behaviour, it is

proven in Eq. (9) that the robustness defined in (4) is a weighted function of standard deviation of the most critical Tsai number evaluated at $b_{s}=b_{a}$. The variance in Eq. (9) can be obtained from

$$
\operatorname{var}\left[\bar{R}\left(\beta_{a}\right)\right]=\sum_{i=1}^{m}\left[\frac{\partial \bar{R}}{\partial \pi_{i}}\left(\beta_{a}\right)\right]^{2} \operatorname{var}\left(\pi_{i}\right)
$$

where $\operatorname{var}\left(p_{i}\right)$ is the variance of the material design parameter, $p_{i}$, defined as a random variable in the vector, $p 2 R^{m}$, and the derivatives are obtained at $b_{s}=b_{a}$ after the sensitivity analysis is performed using the adjoint variable method.

\subsection{Structural reliability assessment}

In the structural reliability analysis of composite structures, the basic random variables, which are assumed to be uncorrelated, define the vector $p\left(p_{1}, p_{2}, \ldots, p_{n}\right)$. Their mean values and variances describe their statistical nature. In this case, the random variables are the elastic and strength properties of the composite laminates of the structure. If the boundary surface of the safety domain is written as

$$
z=\varphi\left(\pi_{1}, \pi_{2}, \ldots, \pi_{n}\right)=0
$$


the values of $p$ belonging to the failure domain will satisfy the inequality:

$$
z=\varphi(\pi)<0
$$

The probability of failure is defined as

$$
P_{f}=P[\varphi(\pi)<0]=\int_{\Omega} f(\pi) d \pi
$$

where $f(p)$ is the joint probability density function of $p, X$ is the failure region, and $u \partial p$ p is the so-called limit state function that separates the design space into failure $(\mathcal{u} \partial p<0 \mathrm{p}$ and safe regions $(u \partial p>0$ p. In this particular case, the formulas presented in Eqs. (7) and (8) for the limit state function can be written as

$$
\varphi(\pi)=\bar{R}-1
$$

From the deterministic point of view the structural failure analysis is associated to the most critical Tsai number defined in Eq. (8) and the composite structure fails at $\bar{R} 1 / 41$. However, in reliability analysis a limit state function must be considered for each point (for example at the Gauss integration points) on structure where the Tsai number is evaluated as defined in Eq. (7). This means that $N_{s}$ limit state functions should be considered and so, reliability analysis becomes very expensive and unpractical. To overcome this difficulty only the limit state associated to the most critical Tsai number $\bar{R}$ will be considered in reliability analysis as proposed in Eq. (14). Since the stress vector is evaluated at discrete points the measure of structural reliability depends on local conditions and on the shape of the failure envelope and so the approximation function, $U$, the first-order approximation to $P_{f}$ can be written as

considered in Eq. (14) is valid as a first design approach.

The distribution of the basic variables, $p_{i}$, and the limit state surface, $u^{\partial} p \mathrm{p}$, are known, and the probability of failure can be employed as a measure of reliability. However, Eq. (13) cannot be evaluated analytically for realistic structures because the calcula-

$$
u_{i}=\frac{\pi_{i}-\bar{\pi}_{i}}{\sigma_{\pi_{i}}}
$$

tion of the integral is too difficult. To avoid this problem, the mo- ment reliability theory is used in this work, namely, the so-called Lind-Hasofer reliability index [13,14]. The advantage of this meth- od is that it is invariant with respect to different failure surface for- mulations in spaces having the same dimension. The Lind-Hasofer method is performed in two steps. The first one consists of project-ing Eq. (11) into the space of standardised variables: $u_{i}$

where $\bar{p}_{i}$ and $r_{p_{i}}$ are, respectively, the mean values and the standard deviations of the basic random variables. The second step measures, in this space, the minimum distance $b$ of the transformed surface

$$
\varphi\left(u_{1}, u_{2}, \ldots, u_{n}\right)=0
$$

Variables $\mathrm{p}_{\mathrm{i}}$ ), is required to lie entirely in the transformed safety domain. On the other hand, considering that the probability density in the standard normal space decays exponentially with the distance from the origin, the point with the maximum probability of failure on the limit-state surface is the point of minimum distance to the origin. From the operational point of view, the search for this point can be formulated as a constrained optimisation problem

$$
\begin{aligned}
& \text { Minimise } \beta(\mathbf{u})=\left(\mathbf{u}^{T} \mathbf{u}\right)^{1 / 2} \\
& \text { Subject to } \varphi(\mathbf{u})=0
\end{aligned}
$$

The assumption that the minimum distance $b$ obtained from the solution of the minimisation problem in Eq. (17) is a measure of reliability is equivalent to the discretisation at one single point of the safety domain boundary, expressed in the space of the standardised variables. This corresponds to the substitution of the hypersurface by the hyperplane passing through the point defined by $\mathrm{u}^{*}$ [3]. By formally introducing a normal probability distribution

$$
P_{f}=\Phi(-\beta)
$$

where $b$ is known as the reliability index, i.e., the minimum distance from the origin to the limit-state surface.

The design point or most probable failure point (MPP), $\mathrm{u}^{*}$, is obtained using an iterative scheme of the Lind-Hasofer method proposed in the Refs. [1,15] and based on gradients evaluated by the adjoint variable method.

\subsection{Algorithm for performance and robustness trade-off}

The RDO is performed by solving the bi-objective problem formulated in Eq. (2), providing an optimal design which is simultaneously reliable and robust. First, two single-objective problems are solved and subjected to all constraints in order to obtain the utopia point. From the minimisation of the performance function defined in Eq. (3), the optimal maximum load for the target reliability index, $b_{a}$, is obtained over the entire domain of ply angle design variable, $a$, as follows:

$$
\begin{array}{ll}
\underset{\lambda, a}{\text { Minimise }} & {\left[\beta_{s}\left(\lambda, a, \mu_{\pi}\right)-\beta_{a}\right]^{2}} \\
\text { Subject to } & \beta_{s}(a, \pi) \geq \beta_{a} \\
& 0 \leq a \leq \frac{\pi}{2}
\end{array}
$$

This is a conventional RBDO inverse optimisation problem and the reliability constraint, $b_{s} \mathrm{\partial x} ; p \mathrm{P}$ ' $2 b_{a}$, is automatically satisfied. To solve the inverse problem (19), an optimisation algorithm based on gradients or evolutionary methods can be implemented. How-ever, for the case with two design variables, load factor $k$ and ply an- gle $a$, a decomposition of the problem is considered, and the following algorithm is proposed: 


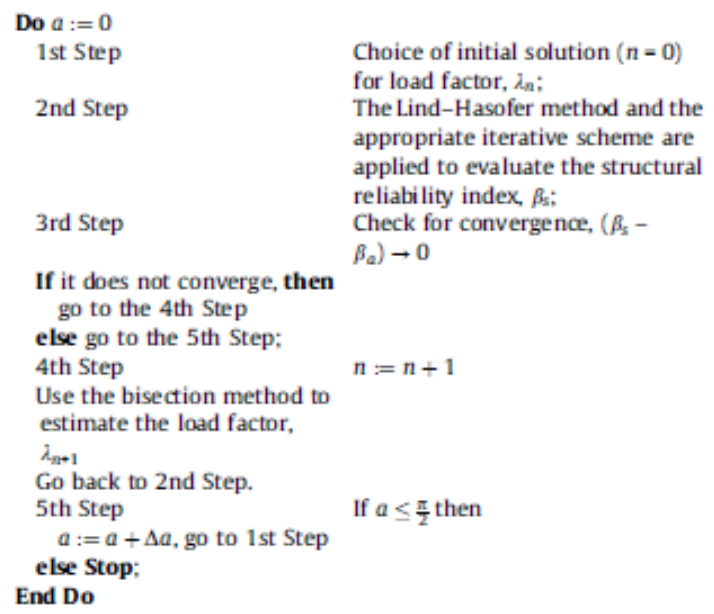

After the minimisation of the performance function given in Eq. (3), the structural reliability index is $\beta_{s} \approx \beta_{a}$, with a prescribed error and the corresponding load vector, $\mathbf{L}\left(\beta_{a}\right)$.।

The second single sub-problem in Eq. (2) minimises the variability of the structural response. This is equivalent to maximising the robustness, established as the coefficient of variation of maximum load $\bar{L} \partial b_{a} \mathrm{P}$, associated with the reliability index target, $b_{a}$, weighted by its nominal value as defined in Eq. (4). This robustness maximisation is performed over the ply angle design variable, $a$, using the optimal solution for maximum load $\bar{L} \circlearrowright b_{a} \mathrm{P}$ obtained from the performance minimisation problem of Eq. (19). Then, the second single-problem is equivalent to

Maximise $\operatorname{ROB}\left(\bar{\lambda}(a), a, \mu_{\pi}\right)$

Subject to $\beta_{s}(a, \pi) \geq \beta_{a}$

$$
0 \leq a \leq \frac{\pi}{2}
$$

where $k \delta a \mathrm{p}$ denotes the maximum load factor associated with $\bar{L} \partial b_{a} \mathrm{p}$.

Since the optimisation problem is composed of two objectives, all optimal solutions belong to the Pareto front. To obtain the trade-off of performance against robustness, two steps are implemented: first, optimal solutions for performance and robustness are normalised relative to its maximum nominal value; second, the minimum distance from the Pareto normed front to the origin of the referential is calculated. The point on the Pareto normed

front associated with the minimum distance can be defined as the best mathematical trade-off between performance and robust-

ness. The corresponding optimal design variable ply angle is

obtained.

\section{Numerical examples}

To test the proposed approach, a clamped cylindrical shell laminated structure made of a E-glass/epoxy (Scotchply 1002, [12]) composite system is considered as shown in Fig. 1. Nine vertical loads of mean value $L_{k}$ are applied along the free linear side (AB) of the structure. This free linear side $(\mathrm{AB})$ is constrained in the $y^{-}$ erence axis, as detailed in Fig. 1. All laminates have the same mean thickness, $h_{k}=0.02 \mathrm{~m}$.

The structural analysis of laminated composite structures is based on the shell finite element model developed by Ahmad[16], with further improvements [17]. This shell element is ob-tained from a 3D finite element using a degenerative procedure. It is an isoparametric element with eight nodes and five freedomdegrees per node based on the Mindlin shell theory. The mean values of the elastic and strength properties of the ply
material used in the laminate construction of the composite structure are presented in Table 1 [12]. The elastic constants of the orthotropic ply are the longitudinal elastic modulus $E_{1}$, the transversal elastic modulus $E_{2}$, the in-plane shear modulus $G_{12}$, the

\section{out-of-plane shear
modulus $G_{13}$}

and $G_{23}$, and the in-plane Poisson's

ratio $m_{12}$. The ply strength properties are the longitudinal strength in tensile, $X$, and in compression, $X^{0}$, the transversal strength in tensile, $Y$, and in compression, $Y^{0}$, and the shear strength, $S$.

To investigate the robust design of the composite structures, the mechanical properties denoted by $(p)$ are the considered random variables. All random variables are non-correlated following a normal probability distribution function defined by their respective mean and standard deviation. The mechanical properties group, $(p)$, includes the following random variables: longitudinal Young's

modulus $E_{1, j}$, transversal modulus $E_{2, j}$, transversal tensile strength

$Y_{i}$, and shear strength $S_{i}$, where subscript $j$ denotes the laminate number. Sixteen mechanical properties are considered as random

parameters with uncertainty in this analysis: $E, E, Y, S$,

$$
1, j \quad 2, j \quad j \quad j
$$

axis direction. The structure is divided into four macro-elements, grouping all elements, and there is one laminate per each macro- 
$j=1, \ldots, 4$. The present study can be extended to other random vari- ables. Different coefficients of variation, C.V.(p), of mechanical prop- erties $(p)$ are tested and the mean values are defined in Table 1.

The constrained minimisation problem given in Eq. (19) is solved, and the maximum allowable load, $L ð b_{a} \mathrm{P}$, on the

element. The laminate distribution of the structure is shown in Fig. 1. The balanced angle-ply laminates with eight layers and the stacking sequence $[+a /+a /-a /-a]_{s}$ are considered in a symmetric construction. Ply angle, $a$, is referenced to the $x$-axis of the ref-

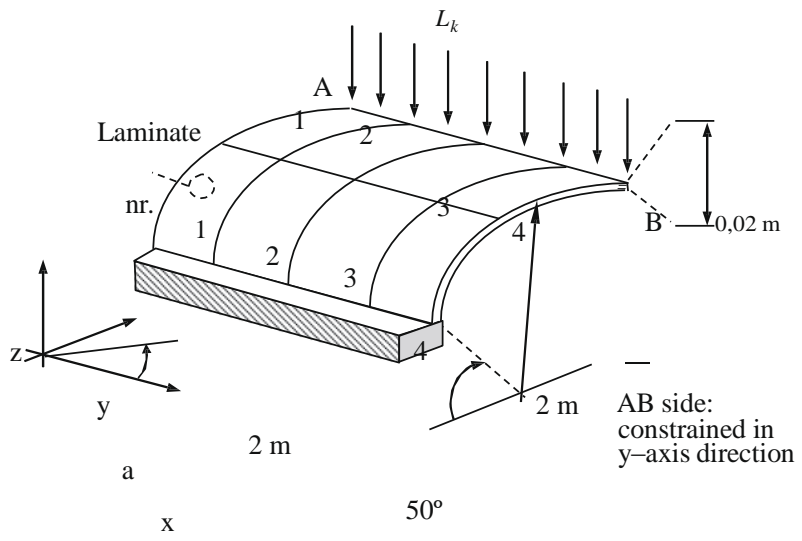

Fig. 1. Cylindrical shell and composite laminates distribution. composite

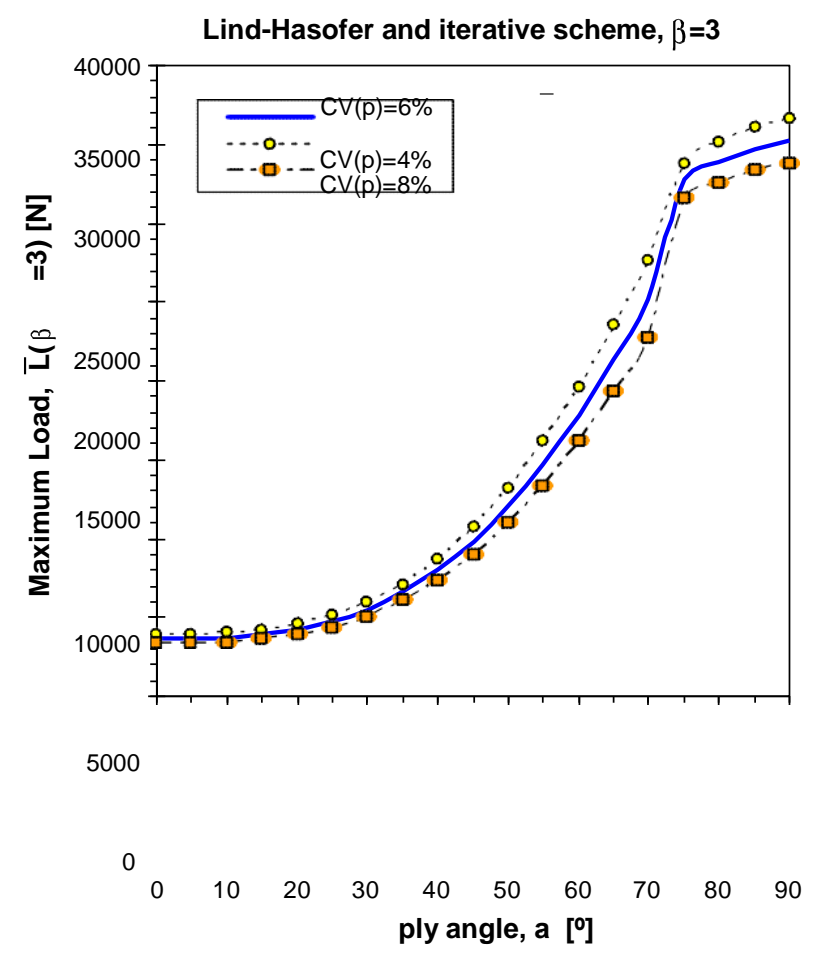

Fig. 2. Performance of structural system measured by maximum load for $b_{a}=3$.

Table 1

Mean value of mechanical properties of unidirectional composite layers.

\begin{tabular}{|c|c|c|c|c|}
\hline Material & $E_{1}(\mathrm{GPa})$ & $E_{2}(\mathrm{GPa})$ & $E_{12}(\mathrm{GPa})$ & $m$ \\
\hline \multirow[t]{2}{*}{ E-glass/epoxy (Scotchply 1002) } & 38.6 & 8.27 & 4.14 & 0.26 \\
\hline & $X ; X^{0}(\mathrm{MPa})$ & $Y ; Y^{0}(\mathrm{MPa})$ & $S(\mathrm{MPa})$ & $q\left(\mathrm{~kg} / \mathrm{m}^{3}\right)$ \\
\hline E-glass/epoxy (Scotchply 1002) & $1062 ; 610$ & $31 ; 118$ & 72 & 1600 \\
\hline
\end{tabular}


structure, considering the prescribed reliability index of $b_{a}=3$, is obtained. This maximum load, as function of ply angle design variable $a$, is plotted in Fig. 2 for different values of the coefficient of variation for the input mechanical properties group $(p)$. The maximum load is more sensitive to variations of the random input variables for ply angles over $45^{\circ}$.

For every kind of random variables, the most probable failure point (MPP) values, depending on ply angle $a$, are presented in Figs. $3-6$, calculated for the coefficient of variation of the input random variables, C.V. $(p)=6$. These figures correspond to the MPP solutions found for the reliability analysis problem defined in Eq. (17) for the particular case of $b_{a}=3$ and the corresponding maximum load shown in Fig. 2. The maximum differences of random variable values at MPP relatively to their mean values are: $8.6 \%$ for longitudinal modulus $E_{1, j}, 9.2 \%$ for transversal modulus $E_{2, j}, 16.3 \%$ for transversal strength $Y_{j}$, and $8.0 \%$ for shear strength $S_{j}$, considering all laminates on the composite structure, i.e., for $j=1, \ldots, 4$. These differences are larger than the coefficient of variation, C.V. $(p)=6$, considered in the input random variables. This means that the
MPP vector $p^{*}$ is very far from vector pof the mean values of random variables. Most of the significant differences for this design problem are associated with ply angles over $45^{\circ}$. Since the MPP is the point with the maximum probability of failure on the limit-state surface, and considering that vector $p$ is not a good approximation of MPP vector $p^{*}$, it can be concluded that reliability analysis must be considered together with robust design optimisation.

The Figs. 3-6 show the influence of the location on structure of the most critical Tsai number $\overline{\mathrm{R}}$. The limit state function associated to $\overline{\mathrm{R}}$ is located on laminate number one $(j=1)$ for ply angle a $\left.20^{\circ} ; 70^{\circ}\right]$ and is located on laminate number four $(j=4)$ for ply angle $a 20^{\circ} ; 70^{\circ}$. The limit state function in Eq. (14) is much more sensitive to the random variables belonging to the laminate where the most critical Tsai number $\overline{\mathrm{R}}$ is evaluated than to the variables associated to other laminates. Thus, the more sensitive random variables are identified by subscripts $j=1$ and $j=4$ in Figs. 3-6 and are the ones that show wider variations in MPP search established by the minimisation problem defined in Eq. (17).

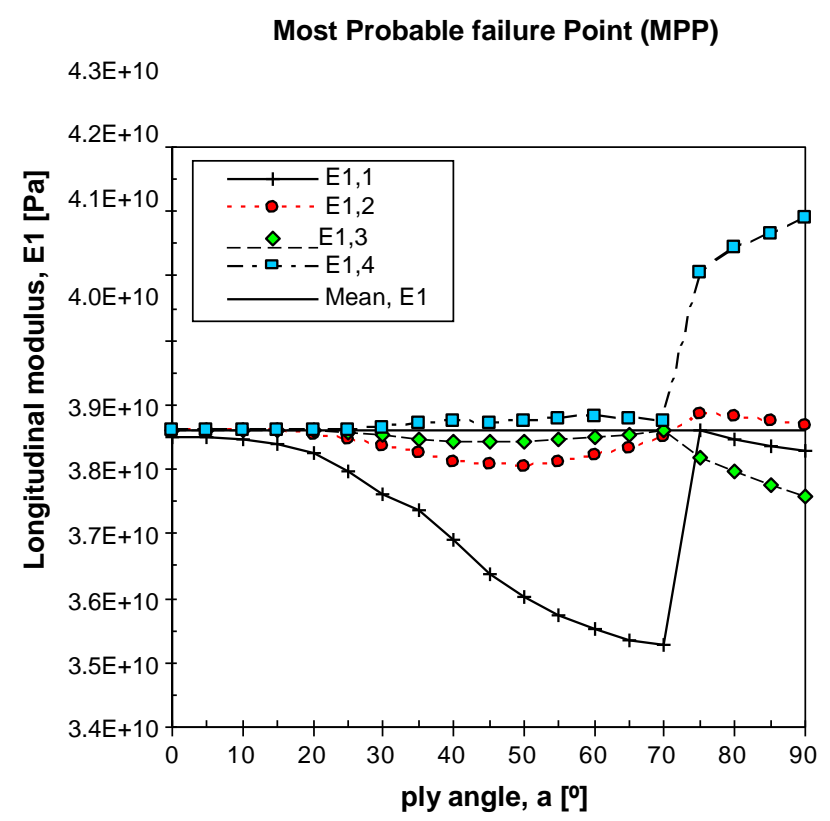

Fig. 3. Variation of MPP value of the longitudinal Young's modulus for $b_{a}=3$.

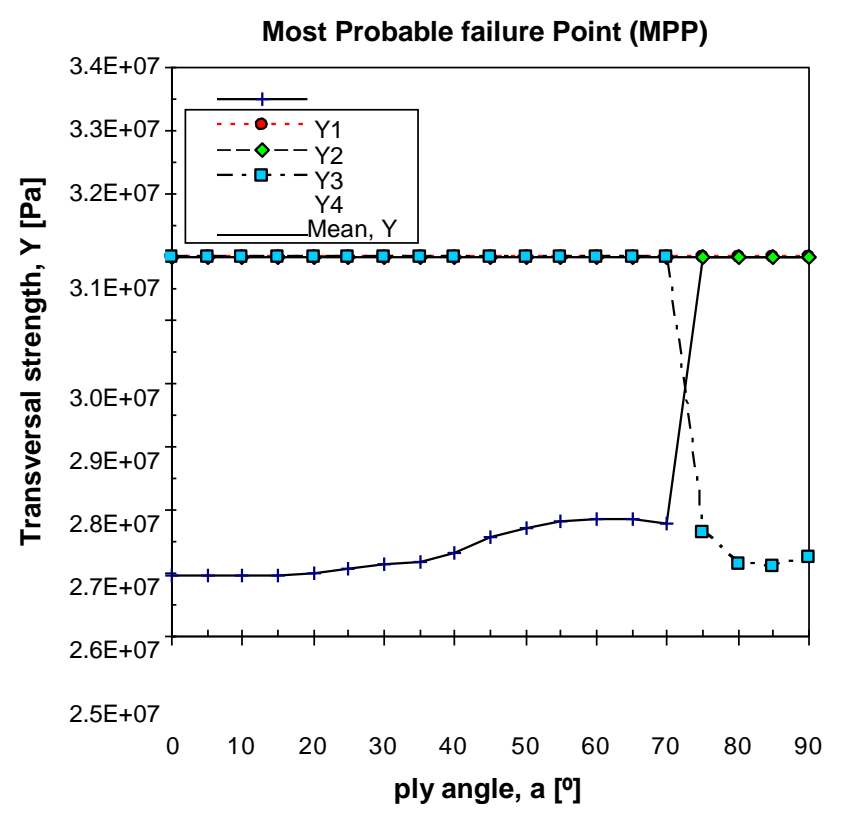

Fig. 5. Variation of MPP value of the transversal strength for $b_{a}=3$.

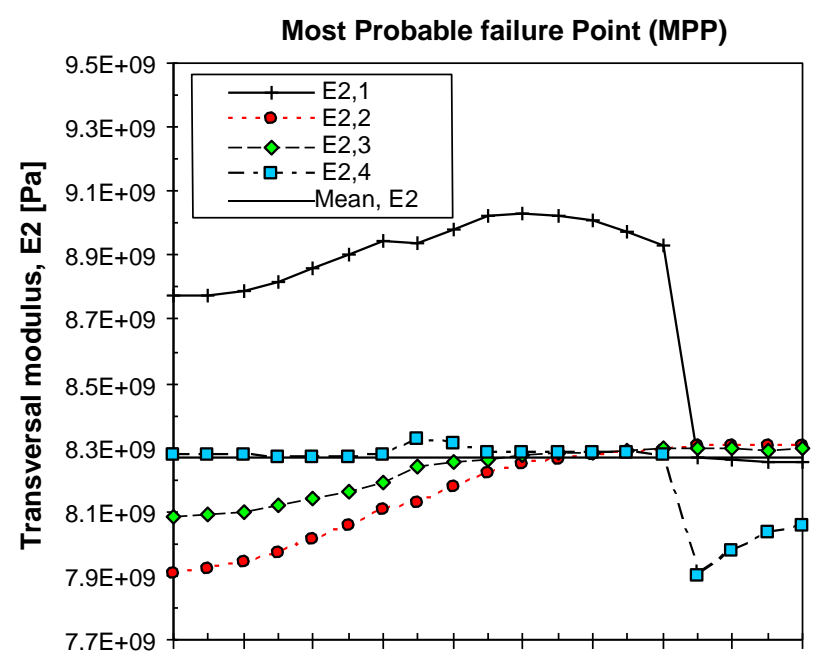

0

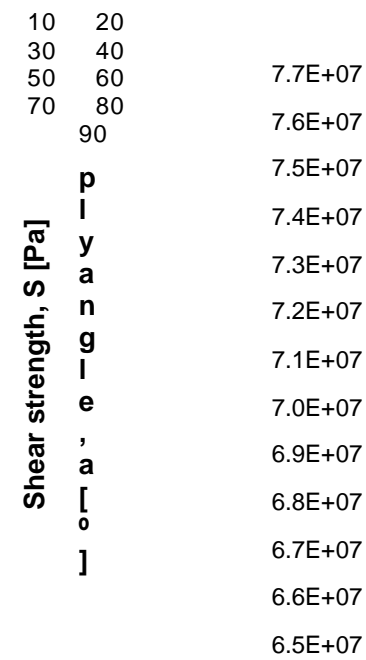


M

$\mathbf{O}$

S

$\mathbf{t}$

P

0

b

Fig. 4. Variation of MPP value of the transversal modulus for $b_{a}=3$.

able failure Point (MPP)

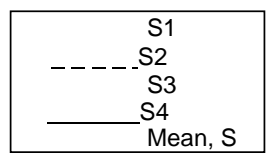

Mean, S

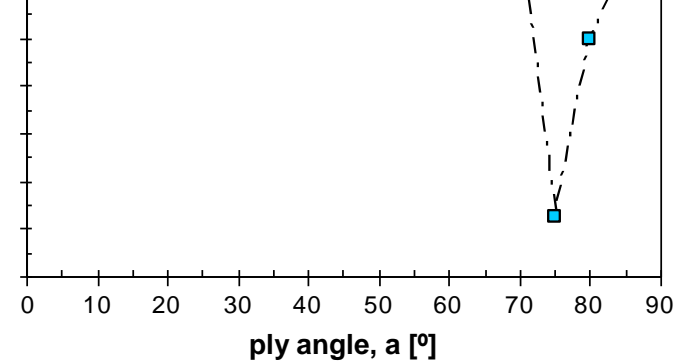

Fig. 6. Variation of MPP value of the shear strength for $b_{a}=3$. 
The results presented in Fig. 2 can be validated using a Bayesian method applied to the inverse problem, i.e., calculating the probability of failure, $P_{f}$, associated with the obtained maximum load,

$L \delta b_{a} \mathrm{P}$, for each ply angle design variable, $a$. The objective of the

Bayesian methods is to use all available knowledge (a priori information) based on a previous experience, intuition, or results obtained in similar previous problems [18]. In this case, the information source is the knowledge of a previous Monte Carlo simulation ( $N^{0}$ simulations, $K^{0}$ failure events). According to the Bayesian techniques, the estimation of the failure probability is based on two distribution functions. The distribution, a priori, is the $\operatorname{Beta} \partial K^{0} \mathrm{p} 1 ; N^{0}-K^{0} \mathrm{p} 1 \mathrm{p}$ law, and the a posteriori distribution is the $\operatorname{Beta} K K \mathbf{p} K^{0} \mathbf{p} 1 ; N-K \mathbf{p} N^{0}-K^{0} \mathbf{p} 1 \mathbf{p}$ law, based on a new Monte Carlo simulation ( $N$ simulations, $K$ failure events). A two sided confidence interval of $P_{f}$ can be calculated with a given confidence $c$. So, the lower $P_{f}^{\text {low }}$ and upper $P_{f}^{u p p}$ limits of the confidence interval of $P_{f}$ can be obtained from,

$$
\int_{0}^{p_{f}^{\text {bow }}} \operatorname{Beta}\left(K+K^{\prime}+1, N-K+N^{\prime}-K^{\prime}+1\right) d P_{f}=\frac{\gamma}{2} \quad p^{\text {low }}
$$

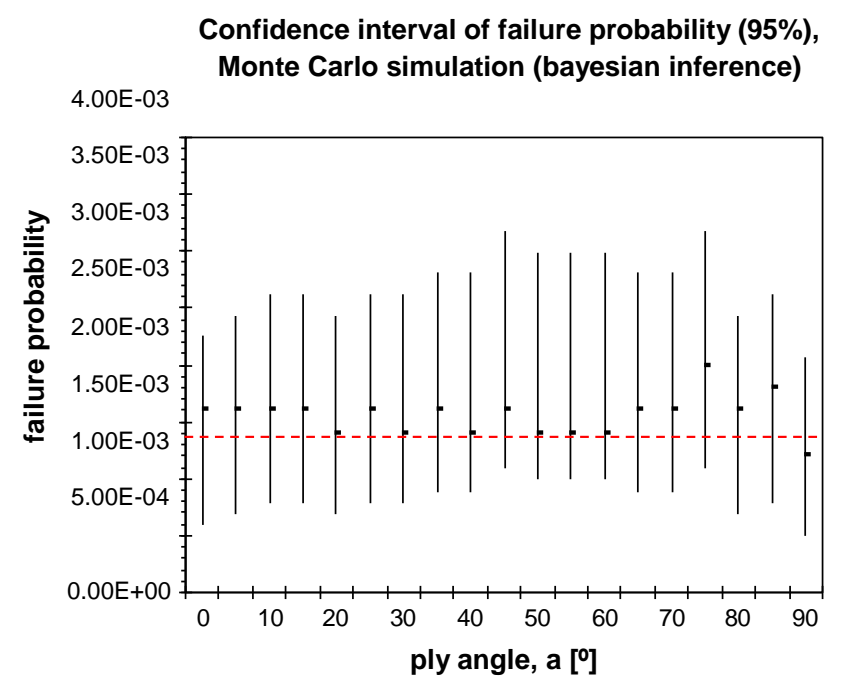

Fig. 7. Confidence interval of failure probability for the structural system.

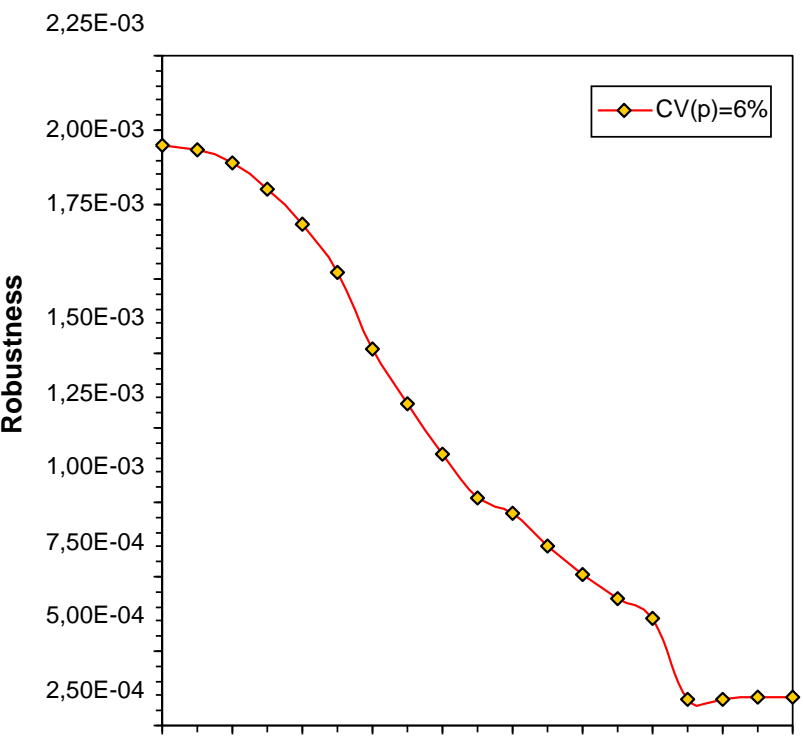

$0,00 \mathrm{E}+00$ $p^{u p p}$

$$
\int_{0}^{p_{f}^{\gamma_{F}^{*}}} \operatorname{Beta}\left(K+K^{\prime}+1, N-K+N^{\prime}-K^{\prime}+1\right) d P_{f}=1-\frac{\gamma}{2}
$$

with, $N_{0}=2000$ first Monte Carlo simulations; $K_{0}=$ failures in the first Monte Carlo simulations; $N=5000$ second Monte Carlo simulations; $K=$ failures in the second Monte Carlo simulations.

In this case, the maximum load is applied to the composite structure for each value of ply angle, $a$, and the Monte Carlo simulation is used, together with Bayesian inference, to confirm the prescribed reliability index of $b_{a}=3$. The confidence interval for $P_{f}$ is shown in Fig. 7, where the horizontal line indicates the failure probability $P_{f}=U(-3)$, calculated using Eq. (18). The horizontal line for $P_{f}=U(-3)$ is inside of the confidence interval, showing the agreement of the results obtained using the Lind-Hasofer method with those from the Monte Carlo simulation together with Bayesian inference.

For every ply angle $a$, the design variable for robustness, as de fined in Eq. (4), is calculated after solving the RBDO minimisation problem presented in Eq. (19). For the proposed numerical exam-

ple, Fig. 8 shows the influence of ply angle $a$. The maximisation problem given in Eq. (20) is solved using the plotted results.

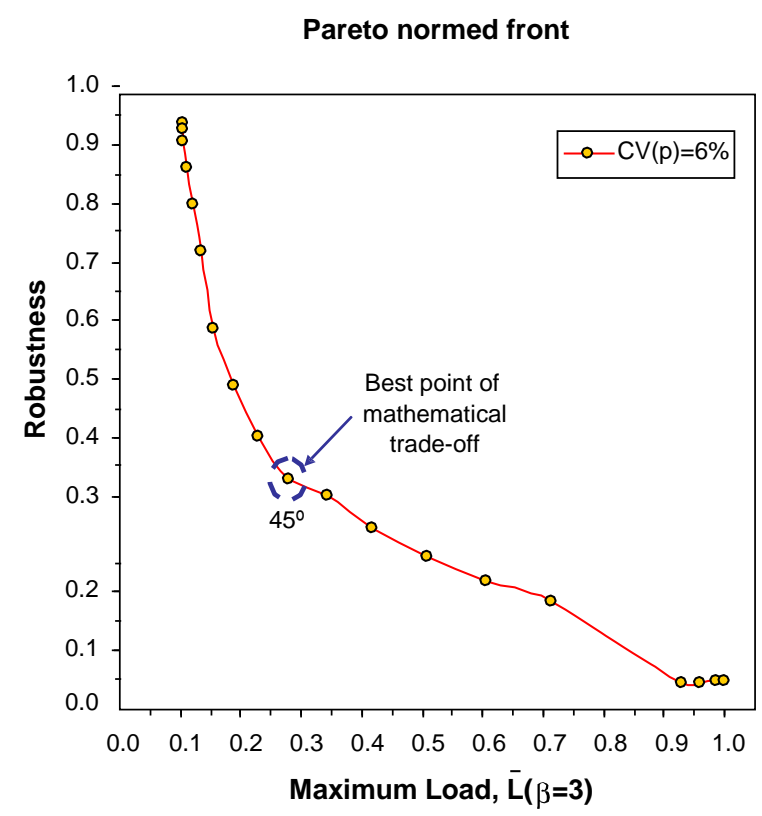

Fig. 9. Pareto curve for robust design optimisation

Table 2

Optimal values for the bi-objective optimisation problem, $b_{a}=3$.

$\begin{array}{rll}\text { Ply angle, } a\left(^{\circ}\right) & \text { Maximum load, } L ð b_{a} \mathrm{P} & \text { Robustness } \\ 0 & 0.102970971 & 1.000000000 \\ 5 & 0.103437757 & 0.992203215 \\ 10 & 0.105961806 & 0.968306559 \\ 15 & 0.111185835 & 0.925590671 \\ 20 & 0.120195227 & 0.862434482 \\ 25 & 0.134468375 & 0.781209716 \\ 30 & 0.155773105 & 0.650483726 \\ 35 & 0.187298885 & 0.552573011 \\ 40 & 0.228160381 & 0.466003676 \\ 45 & 0.278510825 & 0.392310564 \\ 50 & 0.342555546 & 0.364476184 \\ 55 & 0.418716836 & 0.308134663 \\ 60 & 0.506563378 & 0.260146528 \\ 65 & 0.604977157 & 0.219328801 \\ 70 & 0.71235209 & 0.184901369\end{array}$


ply angle, a [으 
A bi-objective optimisation problem based on performance and robustness for angle-ply composite structures with linear elastic behaviour is implemented. At the end of the optimisation processes, the Pareto normed front representing the frontier of the trade-off between the "performance" and "robustness" functions is obtained, as shown in Fig. 9.

The normed values of the objective functions of the bi-objective optimisation problem and the corresponding optimal ply angle are presented in Table 2. According to the considerations made in Section 2.4, the point on the Pareto normed front associated with the minimum distance can be defined as the best mathematical tradeoff between performance and robustness. This point is identified as corresponding to ply angle $a=45^{\circ}$.

\section{Conclusions}

The problem of robust design optimisation (RDO) of composite structures is addressed, considering its performance and variation. In this work, performance is associated with the target imposed on the reliability of the structural system. After solving an inverse problem, the maximum allowable load for a prescribed reliability index is obtained. Robustness is defined by the coefficient of variation of the maximum load weighted by its nominal value. The original bi-objective optimisation problem is decomposed into twosubproblems. First, an inverse reliability-based design optimisa- tion (RBDO) problem is solved, and the maximum allowable load is obtained, by searching over the ply angle domain. Second, based on the solution from the previous problem, maximum robustness is searched over the ply angle domain. At the end of the optimisation processes, the Pareto normed front representing the frontier of the trade-off between the "performance" and "robustness" func- tions is obtained. This bi-objective optimisation is a powerful tool to help designers make decision based on a compromise between performance and robustness.

\section{Acknowledgments}

The authors acknowledge the financial support provided by the Foundation for Science and Technology (FCT), Portugal, through the pluriannual funding 10/225-Unit for Numerical Methods in Mechanics and Structural Engineering.

\section{References}

[1] Conceição António CA, Torres Marques A, Gonçalves JF. Reliability based design with a degradation model of laminated composite structures. Struct Optim 1996;12:16-28.

[2] Boyer C, Béakou A, Lemaire M. Design of a composite structure to achieve a specified reliability level. Reliab Eng Syst Safety 1997;56:273-83.

[3] Conceição António CA. A hierarchical genetic algorithm for reliability based design of geometrically non-linear composite structures. Compos Struct 2001;54:37-47.

[4] Adali S, Lene F, Duvaut G, Chiaruttini V. Optimization of laminated composites subject to uncertain buckling loads. Compos Struct 2003;62:261-9.

[5] Walker M, Hamilton R. A technique for optimally designing fibre-reinforced laminated plates with manufacturing uncertainties for maximum buckling strength. Eng Optim 2005;37:135-44.

[6] Cacuci DG. Sensitivity and uncertainty analysis. Theory, Vol. 1. Boca Raton (FL): Chapman \& Hall/CTC Press; 2003.

[7] Helton JC, Davis FJ. Survey of sampling-based methods for uncertainty and sensitivity analysis. Reliab Eng Syst Safety 2006;91:1175-209.

[8] Huang B, Du X. Analytical robustness assessment for robust design. Struct Multidiscip Optim 2007;34:123-37.

[9] Conceição António C, Hoffbauer LN. From local to global importance measures of uncertainty propagation in composite structures. Compos Struct 2008;85:213-25.

[10] Yang J, Liew KM, Kitipornchai S. Stochastic analysis of compositionally graded plates with randomness under static loading. Int J Mech Sci 2005;47:1519-41.

[11] Choi JH, Lee WH, Park JJ, Youn BD. A study on robust design optimization of layered plate bonding process considering uncertainties. Struct Multidiscip Optim 2008;35:531-40.

[12] Tsai SW. Composites design. Think composites. USA: Dayton; 1987.

[13] Hasofer AM, Lind NC. Exact and invariant second-moment code format. J Eng Mech Div, ASCE 1974;100:111-21.

[14] Melchers RE. Structural reliability analysis and prediction. Chichester: Wiley; 1999.

[15] Conceição António CA. Optimization of structures using composite materials made of polymeric matrix. PhD thesis, Faculty of Engineering, University of Porto, Portugal; 1995 (in Portuguese).

[16] Ahmad S. Curved finite elements in the analysis of solid, shell and plate structures. $\mathrm{PhD}$ thesis, University College of Swansea, UK; 1969.

[17] Figueiras JA. Ultimate load analysis of anisotropic and reinforced concrete plates and shells. PhD thesis, University College of Swansea, UK; 1983.

[18] Guérin F, Barreau M, Charki A, Todoskoff A. Bayesian estimation of failure probability in mechanical systems using Monte Carlo simulation. Quality Technol Quant Manage 2007;4:51-70. 